\title{
Visualization of the constellation of protons in the product-inhibited state of human manganese superoxide dismutase

\author{
Jahaun Azadmanesh ${ }^{1}$, WIIliam Lutz ${ }^{2}$, Leighton Coates ${ }^{3}$, Kevin Weiss ${ }^{4}$, Gloria Borgstahl ${ }^{5}$
} \\ ${ }^{1}$ Univ. Nebraska Medical Center ${ }^{2}$ University of Nebraska Medical Center, ${ }^{3}$ Oak Ridge National Laboratory, ${ }^{4}$ Oak Ridge National Laboratory, ${ }^{5}$ The Eppley Inst For Cancer Res \\ jahaun.azadmanesh@unmc.edu
}

Oxidoreductases are integral to human vitality though most of their mechanisms are unclear due to the difficulty in observing the protons that are used as chemical tools to drive the electron transfers in a process called concerted proton-electron transfer (CPET). Human mitochondrial manganese superoxide dismutase (MnSOD) is a prominently studied oxidoreductase in clinical research due to its central role in the oxidative stress response where it uses CPETs to convert superoxide (O2•-) into either oxygen (O2) or hydrogen peroxide (H2O2). Like many other oxidoreductases, the protonation states and proton transfers (PTs) needed to facilitate the ETs are unclear. Our group's recent work published in Nature Communications on room temperature, wildtype Mn3+SOD, and Mn2+SOD structures revealed portions of the CPET mechanism including an unusual back-and-forth amide deprotonation-and-protonation at Gln 143 that is proximal to the Mn ion. Neutron diffraction's ability to resolve proton positions while being inert to the electronic states of metals and other paramagnetic centers (unlike X-rays) is indispensable for filling the gaps of mechanistic knowledge that are present for oxidoreductases.

Another layer of complexity is provided by human MnSOD's unique feature of product-inhibition that limits the output of $\mathrm{H} 2 \mathrm{O} 2$. The capacity to become inhibited by its product has shown to be a necessity for the normal functioning of human cells, and if perturbed, leads to dramatic cell cycle effects. MnSOD uses this alternative pathway $50 \%$ of the time during steady-state conditions and is thought to be characterized by decoupling ETs and PTs into separate kinetic steps. O2 - binds and abstracts an electron from $\mathrm{Mn} 2+$ without occurrence of PTs to yield a complex of (Mn3+-O22-) incapable of performing ETs. To relieve inhibition at the active site, kinetic and mutagenesis studies suggest an alternative array of PTs is used to protonate peroxide anion to hydrogen peroxide. This demonstrates the activity of an oxidoreductase is regulated by a chemical decision to perform electron and proton transfers in concert though the basis of such chemistry has not been experimentally explored.

Comparison of other human MnSOD point mutants and MnSOD from other species suggests that the precise position of Gln143 affects the extent of product inhibition. We obtained neutron diffraction data to $2.3 \AA$ resolution on a perdeuterated Trp161Phe MnSOD crystal cryotrapped with the peroxide ligand. The Trp161Phe slightly perturbs the packing at the active site leading to a shift of the Gln143 position and helped enrich the productinhibited complex. The position of the peroxide molecule coincides with the X-ray counterpart. From the neutron diffraction data, X-ray absorption spectra, and quantum mechanical calculations, we have derived (1) the chemical significance of the Gln143 position for inhibition, (2) the protonation and electronics conditions leading to product inhibition, and (3) the proton transfer array used to protonate the peroxo-Mn complex and relieve inhibition. The work sets a foundation for investigating CPET mechanisms of oxidoreductases that are largely unknown. 